\title{
Neural Glioblastoma
}

National Cancer Institute

\section{Source}

National Cancer Institute. Neural Glioblastoma. NCI Thesaurus. Code C111693.

A molecular subtype of glioblastoma characterized by the expression of the neural

markers NEFL, GABRA1, SYT1, and SLC12A5. 